\title{
El transporte masivo y su impacto en la movilidad urbana de Pachuca
}

\section{Mass transportation and its impact on urban mobility in Pachuca}

\begin{abstract}
Laura Myriam Franco-Sánchez*
Carlos Mejía-Reyes*

Recibido: febrero 09 de 2021.

Aceptado: agosto 05 de 2021.

\section{Resumen}

Tanto la situación general del funcionamiento del transporte público como su impacto en las condiciones de movilidad de la población han sido motivo de intervenciones gubernamentales en la Zona Metropolitana de Pachuca (ZMP). Así, se implementó el transporte masivo denominado Tuzobús con el fin de mejorar el servicio de transporte público en la ciudad y sus áreas conurbadas; sin embargo, se han evidenciado importantes limitaciones debido a modificaciones en rutas y a una nueva reconfiguración del transporte público en la zona, donde se eliminaron otras opciones de traslado. Esta investigación plantea conocer la percepción de la población pachuqueña usuaria del transporte masivo Tuzobús. Dentro de los hallazgos, principalmente, se presentan problemas relacionados con las tarifas, el tiempo utilizado y la calidad del servicio.
\end{abstract}

Palabras clave: percepción, movilidad urbana, transporte.

Abstract

The general situation of the operation of public transport and its impact on the mobility conditions of the population have been the reason for government interventions in the Metropolitan Area of Pachuca (ZMP), from which the implementation of mass transport known as Tuzobús emerged as a way to improve the public transport service in the city and its conurbation areas. However, situations have been observed in which important limitations are evident, due to modifications in routes and a new reconfiguration of public transport in the area, where other options to travel were eliminated.

For this reason, this document proposes to know the perception of the Pachuco population that uses the Tuzobús mass transport. Among the findings, mainly, there are problems related to the rates, the time used, and the quality of the service.

Keywords: perception, urban mobility, transport.

*Universidad Autónoma del Estado de Hidalgo, México. Correos electrónicos: myriam_franco@hotmail.com,
carlos_mejia7563@uaeh.edu.mx




\section{Introducción}

La movilidad urbana es un desafío para las ciudades, ya que el desplazamiento de millones de personas es una variante en su calidad de vida y afronta retos de gobernabilidad y contaminación. Las transformaciones territoriales en las que están inmersos los espacios metropolitanos permiten alcanzar un número de destinos más dispersos y alejados del hogar para realizar un mayor número de actividades (Gutiérrez y García, 2005).

Tanto la situación general del funcionamiento del transporte público como su impacto en las condiciones de movilidad de la población han sido motivo de intervenciones gubernamentales y no es la excepción la Zona Metropolitana de Pachuca (ZMP), ${ }^{1}$ donde se implementó el transporte masivo denominado Tuzobús, como una forma de mejorar el servicio de transporte público en la ciudad y sus áreas conurbadas. Sin embargo, después de su puesta en marcha, se han observado importantes limitaciones; por ejemplo, las rutas de transporte, independientemente de estar o no relacionadas con los ramales del Tuzobús, fueron modificadas, originando una reconfiguración del transporte público en la zona.

Esta investigación plantea como objetivo conocer la percepción de la población pachuqueña usuaria del transporte masivo Tuzobús, teniendo como premisa que, pese a la necesidad de tener un transporte ordenado y eficiente, no ha cumplido en su totalidad la expectativa de los usuarios. Es indispensable resaltar que los problemas de este sistema de transporte no sólo responden a lógicas de implementación, sino también de gestión, las tarifas; además, el reparto de ganancias entre el gobierno y el sector privado parece no estar relacionado con la búsqueda de la mejora que marcan tanto el Plan Nacional de Desarrollo (Secretaría de Gobernación, 2020) como el Plan Estatal de Desarrollo (Gobierno del Estado de Hidalgo, 2020).

\section{Metodología}

Para la realización de este estudio, se obtuvo información de dos encuestas levantadas por el Instituto Nacional de Estadística e Informática (INEGI): la Encuesta Intercensal 2015 y la Encuesta Nacional de Calidad e Impacto Gubernamental 2017 (INEGI, 2015; 2017). La primera tiene como objetivo principal conseguir datos sociodemográficos de la población en México; por primera vez, incluye preguntas para conocer la movilidad educativa, así como los traslados por motivos de trabajo que ya habían sido retomados desde el Censo del 2000. Además, el tiempo de traslado y el medio de transporte que utilizan estas personas para llevar a cabo sus movimientos. Por tanto, esta información permitió allegarnos a una valoración de lo que implica la movilidad, considerando los viajes realizados de manera lineal entre el lugar de origen y el de destino que frecuentemente realiza la persona.

\footnotetext{
${ }^{1}$ La integran los municipios de Epazoyucan, Mineral de la Reforma, Mineral del Monte, Pachuca de Soto, San Agustín Tlaxiaca, Zapotlán de Juárez y Zempoala.
} 
Por su parte, la Encuesta Nacional de Calidad e Impacto Gubernamental (INEGI, 2017) se tomó como base para conocer el grado de satisfacción que tienen la población de Pachuca sobre el sistema de transporte Tuzobús; uno de sus objetivos principales es medir la satisfacción de los usuarios de servicio público básicos durante 2017; para ello, se fundamenta en la norma europea sobre calidad de transporte público desde la perspectiva del usuario (UNE-EN 13816):

retoma los criterios de: disponibilidad, accesibilidad, información sistemática, tiempo de traslado, comodidad y seguridad, por lo que considera que un transporte de calidad debe: subir pasajeros sólo en paradas oficiales, contar con tablas de horarios y corridas, transcurrir poco tiempo entre unidades, tener suficiente espacio para viajar cómodo y suficientes rutas para que los usuarios lleguen a su destino, estar limpios y en buenas condiciones. Además, los pasajeros deben recibir un trato respetuoso y amable por parte del operador (INEGI, 2017: 44).

La información captada en el cuestionario de esta encuesta permitió conocer la importancia de este medio de transporte y su valoración. Finalmente, se aplicaron 15 entrevistas semiestructuradas a los usuarios a lo largo de la línea del Tuzobús, la cual parte del centro histórico de la ciudad y recorre parte de ésta para pasar, finalmente, sobre el boulevard Felipe Ángeles. La intención es explicar el fenómeno, no de representar el problema del funcionamiento del Tuzobús.

Las entrevistas mostraron la percepción del uso de este medio de transporte por medio de la narración directa de los actores; se entrevistó a amas de casa, estudiantes y empleados, permitiendo caracterizar y entender mejor el funcionamiento de este servicio; se consideraron cuatro variables fundamentales para evaluar: la cobertura, la accesibilidad, la utilización y la calidad.

Así, se identifican los principales patrones de desplazamiento, abriendo la discusión respecto al tema de la movilidad urbana y su relación con el transporte masivo. El estudio pone en evidencia que el sistema de transporte masivo en la Zona Metropolitana de Pachuca ha traído consigo grandes problemas en la movilidad de la ciudad; para ello, se presentan datos y entrevistas a los usuarios. La metodología de análisis se obtuvo con información de las encuestas mediante el análisis estadístico descriptivo; asimismo, refuerza los hallazgos encontrados en la información institucional con la percepción de los usuarios.

\section{La movilidad urbana y el transporte}

La urbanización y la metropolización han propiciado la generación de flujos migratorios regionales y, a su vez, su redistribución hacia las ciudades intermedias, pues ofrecen condiciones que se perciben como una alternativa a la crisis urbana de las grandes ciudades. 
Además, han permitido cambios en los procesos socioespaciales referentes a los movimientos de población, por lo que han conllevado a que los sistemas de movilidad sean cada vez más complejos.

En 2020, se identificaron 1,934 metrópolis en el mundo, en las cuales habitaban poco más de 300,000 personas que representaban el 60\% de la población urbana del mundo. Hoy en día, un tercio de la población mundial vive en metrópolis, es decir, alrededor de 2.59 mil millones de personas. Además, 34 metrópolis han superado los 10 millones de habitantes, mientras que 51 tienen una población de 5 a 10 millones; 494 de 1 a 5 millones; y 1,355 de 300,000 a 1 millón (ONU-HABITAT, 2020).

En América Latina y México, el desarrollo urbano se ha manifestado de diversas formas, lo que ha traído consigo consecuencias permeables a la movilidad, lo más significativo es la ubicación espacial de las personas en relación con sus centros de trabajo, lugar de estudios y entretenimiento. Lo más común es que los pobladores de bajos ingresos ocupen regiones periféricas en condiciones desfavorables, donde el valor del suelo es mínimo, permitiéndoles instalarse y construir su hogar en condiciones muy complicadas y austeras.

Conjuntamente, en dichos lugares la oferta de servicios públicos y centros de empleo son escasos e, inclusive, llegan a ser nulos, lo cual implica que la población tenga que recorrer grandes distancias a diario sólo para llegar a su lugar de trabajo; además, los trayectos en sí conllevan un considerable aumento en el costo del transporte público. Aunado a ello, el expansivo crecimiento poblacional en las ciudades latinoamericanas ha implicado el incremento de procesos de urbanización, los cuales carecen de planeación y diseño, cuyos efectos impactan drásticamente tanto en el funcionamiento y en la fluidez de la movilidad de estos centros urbanos como en la ausencia de una cultura vial para peatones y para automovilistas, lo cual ha orillado a un uso incorrecto de la infraestructura existente en las ciudades empeorando el estado de la movilidad urbana.

Las ciudades son un espacio natural para el estudio de la movilidad; las personas se trasladan para trabajar, ir a sus hogares o realizar otros hábitos cotidianos; las urbes se transforman con el paso del tiempo; se observan como una expansión que cada día crece y alberga a distintos tipos de personas; es decir, una colección de distintos sistemas que interactúan entre sí.

Los cambios que se han generado en las ciudades latinoamericanas han modificado las estructuras de la población que requieren una nueva manera de enfrentar la difícil tarea del planear y diseñar las ciudades existentes y nuevas. Estos procesos no son ajenos; en el pasado, en Europa, se gestaron los mismos problemas y desafíos que enfrentan las ciudades actuales (Jans, 2017). Es fundamental conocer cómo se ha dado el crecimiento urbano en las ciudades latinoamericanas, el cual se ha basado en la dispersión, cuyo factor ha sido decisivo en la generación de las deficiencias de la movilidad urbana en muchas ciudades o 
metrópolis. La ZMP no ha sido ajena a este proceso, pues ha seguido este mismo patrón, acompañada del incremento del parque vehicular, la nula regulación del transporte, la ausencia de políticas y de normas de regulación ordenamiento territorial y planificación urbana.

La movilidad urbana implica un cúmulo de desplazamientos de personas y mercancías dentro de una ciudad; para ello, se utiliza tanto transporte público como privado, ya sea motorizado o no; por ejemplo, en el primer caso: automóviles particulares, tranvías, metro, combis, autobús, taxis, entre otros; y en el segundo: caminar, bicicleta, etcétera.

El término movilidad urbana va más allá de lo que involucra la palabra transporte, pues no sólo hace hincapié en la dinámica de los desplazamientos (cantidad, frecuencia, infraestructura y medios de transporte), sino también considera aspectos socioeconómicos, espaciales, edad, género y estatus laboral. Por tanto, se define como aquellos desplazamientos de personas dentro de una ciudad con el objetivo de minimizar la distancia existente que separa los espacios, los cuales se llevan a cabo caminando o en diferentes medios o sistemas de transporte: bicicleta, coche, autobús, metro, etcétera.

Además, esta movilidad describe a todas las demás de diferente escala espacial y temporal realizadas en el ámbito de un sistema urbano, pues hay referencias a la movilidad cotidiana vinculada a la migración pendular entre el hogar y el trabajo $u$ otras actividades; a la residencial, relacionada con el mercado inmobiliario; y a la profesional, vinculada al mercado de trabajo (Korsu, 2010). Asimismo, se define como un elemento que determina tanto la productividad económica de la ciudad y la calidad de vida de sus habitantes como el acceso a servicios básicos de salud y educación (Ciudadanía metropolitana, 2018).

La movilidad es un tema trascendente en las investigaciones urbanas y de transporte debido a su relación con los movimientos de población diarios que implican la separación espacial de las funciones de la vida diaria: vivienda, trabajo, abasto y recreación, condiciones resultantes de la estructura urbana, las diferencias, las desigualdades sociales, económicas y espaciales que se dan en un territorio determinado, siendo una característica de las aglomeraciones urbanas (Arango, 2010).

Por tanto, la movilidad presenta una relación estrecha con el transporte, además de relacionarse con la ubicación de las fuentes de empleo, ya que indirectamente influye sobre la decisión de localización residencial. Por ello, es de interés conocer cómo se presenta la movilidad de las personas, ya sea individual o colectiva. La calidad de la movilidad y accesibilidad está estrechamente vinculada con la ordenación y la planeación de los sistemas de transporte, ya sea mediante inversión pública o privada.

Las ciudades se caracterizan por ser espacios dinámicos regidos por diversos sectores socioeconómicos, los cuales actúan dentro de una estructura económica urbana, expresándose a través de la forma y de la estructura propia. Sin embargo, su crecimiento es 
desordenado mostrando una estructura urbana discontinua e irregular carente de articulación funcional, dificultando la movilidad de la población en un ir y venir cotidiano (Basant, 2018). La movilidad involucra un impacto importante en las sociedades actuales donde ha sido auspiciada por los diferentes avances tecnológicos a nivel mundial, los cuales han acortado las distancias, por lo que involucra el desplazamiento de personas y mercancías, pero también conlleva el movimiento de la información misma. Por tanto, en cuanto más desarrollado sea un país, requerirá mayor movilidad.

De acuerdo con Negrete (2018), la movilidad urbana se determina por la productividad económica de las ciudades facilitando a los ciudadanos desplazarse a sus centros de trabajo, de abastos de alimentos, de educación, salud, culturales y recreativos. Asimismo, dicha movilidad tiene una relación estrecha con el transporte urbano, el cual es un eje del bienestar de la sociedad.

Por consiguiente, la movilidad mantiene una estrecha relación con el funcionamiento de los sistemas de transporte; la infraestructura vial que mantiene una injerencia directa a los desplazamientos de las personas. Hoy en día, hablar de movilidad implica entender la construcción social del territorio, donde las dimensiones: urbana, socio-ambiental y de gestión están presentes dando una visión de la movilidad como un elemento articulador territorial y definiendo la perspectiva del uso de la ciudad y la construcción del territorio. Así, la movilidad no sólo involucra desplazamiento, sino que en ella están inmersas las condiciones que facilitan o entorpecen su realización; deja en sí, un concepto flexible y multidimensional que abarca el proceso de habitar y acceder, derechos sociales y procesos de diferenciación socio-espacial, los cuales deben ser plasmados en la planeación y gestión pública (Cruz-Muñoz, 2018).

La movilidad tiene profundas implicaciones para la planificación y gestión urbana, principalmente a través de la generación de nuevas necesidades de provisión de medios de transporte y servicios específicos, no sólo en el lugar de residencia, sino también en el de trabajo y estudio; es importante reconocer que hoy en día los espacios de movilidad son esenciales para la vida (Jirón Martínez, 2015). Los factores estructurales como la realidad económica, la infraestructura del transporte y la dinámica del mercado del suelo determinan las condiciones de movilidad urbana para la población de la ciudad.

En los países en vías en desarrollo, el sector transporte es de gran relevancia ante los procesos de crecimiento urbano, así como en las relaciones sociales y económicas, pues se considera un problema multifacético y complejo, donde la comunicación vial ejerce influencia en el proceso de expansión de las ciudades (Comisión Asesora Presidencial Pro Movilidad Urbana, 2014). El transporte, la circulación y la accesibilidad se relacionan estrechamente con la movilidad y, a su vez, con la forma y estructura de la ciudad. 
La movilidad cotidiana está ligada al comportamiento del transporte; la realidad como se presenta en cada país o ciudad influye directamente en los movimientos de las personas, lo cual ha eliminado los impedimentos para su crecimiento y ha contribuido a la desconcentración de los centros urbanos hacia las zonas periféricas. Los patrones actuales de cómo construir una ciudad abocan a la fragmentación del territorio, por lo que existe una fuerte dependencia del vehículo privado para los desplazamientos (Avellaneda y Lazo, 2011).

La movilidad es un elemento medular en las sociedades urbanas; involucra desplazamientos que permiten la comunicación, el desempeño de la actividad económica y las relaciones sociales; es una necesidad que tienen las personas para allegarse de los bienes y servicios indispensables para su vida; es un derecho humano que incluye compromisos y obligaciones por parte de los gobiernos; por tanto, las políticas de movilidad, bajo un enfoque de derechos humanos, deben dirigirse al cumplimiento de éstas considerando la disponibilidad, la accesibilidad y la calidad como los componentes principales de este derecho (ITDP, 2013).

Hablar de movilidad implica el movimiento de bienes y personas y no propiamente al vehículo que es ocupado para realizar estos traslados; los viajes derivados de la demanda de transporte llevan consigo un valor que se traduce cuando las personas se conectan a sus destinos y sus costos deben ser reducidos significativamente (Vadillo, 2019). Las políticas públicas han desatendido la movilidad urbana en las ciudades latinoamericanas; en estos lugares, las poblaciones que usan bicicleta, transporte público o simplemente caminan son aquellas que no cuentan con los recursos suficientes para acceder a otro modo de transporte; de acuerdo con lo señalado por Pérez (2015), en una encuesta realizada a mil personas que se encontraban caminando en la Ciudad de México se identificó que pertenecían en su mayoría a clases populares y medias y sus desplazamientos eran pendulares de su casa al trabajo usando para ello el transporte público para moverse por la ciudad.

La movilidad urbana se relaciona indiscutiblemente con el transporte, los deseos que tienen las personas de viajar de una zona a otra dentro de la ciudad, consecuencia de su interacción social y económica. Así, en una zona de la ciudad, se habita; en otra, se trabaja; y en otra, están los centros de diversión y esparcimiento, etcétera. De acuerdo con Bazant (2018), al habla de movilidad urbana se reflexiona sobre las diferentes rutas de deseos de desplazamiento de las personas a través de la ciudad, por lo cual los tipos de movilidad urbana estarán definidos en razón a su forma y estructura; los desplazamiento de las personas se llevan a cabo mediante diferentes arterias de comunicación: avenidas principales, secundarias, distribuidores viales, que vinculan de manera funcional las diversas zonas de la ciudad, como sectores habitacionales, medios y residenciales o extensas zonas de asentamientos populares con los más concurridos destinos que, principalmente, son los centros y subcentral urbanos. 
El transporte va de la mano con los avances tecnológicos y en particular está ligado a los medios urbanos. Los grandes adelantos de las últimas décadas, en combinación con procesos de reestructuración de los modelos productivos, han hecho del transporte el principal factor de procesos como el urban sprawl y la periurbanización (Suárez y Delgado 2010). El tipo de transporte está ligado al nivel de ingresos; personas de bajos ingresos se caracterizan por tener una movilidad peatonal, en bicicleta o, en su caso, en transporte público; quienes tienen ingresos más altos requieren movilizarse por medio del automóvil (Alcántara, 2010). Así, el transporte público juega una parte fundamental en la movilidad cotidiana; en las ciudades medias se han introducido transporte tipo Tuzobús para aliviar la saturación de usuarios que ocupan este medio.

\section{El transporte masivo en algunas ciudades latinoamericanas}

El transporte masivo tiene sus antecedentes en América; en 1972, Curitiba, Brasil, fue la primera ciudad que manejó este tipo de transporte, y de ahí se ha extendido a otras ciudades latinoamericanas, como el transmilenio en Bogotá, Colombia; el metrobus, el Mexibús, el acabus, entre otros, en México; el metropolitano en Lima, Perú; el metrovía en Guayaquil, Ecuador; y el transmetro en Guatemala. Aunque las características en cada uno de estos países han tenido variantes en infraestructura, vehículos y servicio, todos tienen como objetivo final obtener una mayor calidad en la movilidad manteniendo un elevado nivel de satisfacción en los usuarios y reduciendo tiempos de traslados, cómodos, confiables, con mínimos tiempos de espera en paradas y tiempos de permanencia (Zamora et al., 2013).

Este transporte en Curitiba se considera una innovación enfocada al desarrollo económico y social de la región metropolitana; mantiene una red de $790 \mathrm{~km}$ de extensión cubriendo la demanda de aproximadamente del 75\% de personas del Área Metropolitana de Curitiba. Su estructura se basa en vías exclusivas en cada corredor que se encuentran en el centro de un sistema "trinario": tres vialidades paralelas y las dos hileras de manzanas intercaladas. Este sistema es conocido y reconocido mundialmente por ser pragmático, integrado, eficiente y rentable, aunado que su uso beneficia al medio ambiente dada la disminución de uso del automóvil (Mendoza y García, 2013).

El transmilenio en Bogotá es una propuesta cuyo objetivo fue mejorar la movilidad urbana de la ciudad y mantener una alta ocupación. Su objetivo primordial es mejorar la calidad de vida y la productividad de la ciudad; se extiende alrededor de $87 \mathrm{~km}$; las vías troncales son los carriles centrales de las principales avenidas de la ciudad, los cuales se acondicionan para soportar el paso de los buses y se separan físicamente de los carriles de

\footnotetext{
${ }^{2}$ Describe la expansión de poblaciones humanas lejos de las áreas urbanas centrales en comunidades de baja densidad, monofuncionales y usualmente dependientes del automóvil, en un proceso llamado suburbanización (Karakayaci, 2016).
} 
uso mixto, disponibles para circulación de vehículos particulares, camiones, taxis, etcétera. Desde el inicio de su operación, ha mostrado beneficios y potencialidades importantes; han disminuido los índices de duración de los viajes, así como los de contaminación por gas y accidentes de tráfico (Zamora et al., 2013: 110-111).

Sin duda, estos sistemas de transporte han tenido éxito en los países donde se han llevado a cabo; en México, se ha implementado en algunas ciudades capitales; por ejemplo, el metrobus de la Ciudad de México cuenta con siete líneas, una cobertura de 125 kilómetros y 239 estaciones; y el Mexibús en el Estado de México se integra por tres líneas, nueve estaciones y una extensión de 31 kilómetros, con lo cual ha tratado de dar cobertura a la demanda de transporte de la población; sin embargo, ha tenido diferentes problemas en su operación por falta de unidades y por sobre saturación en la mañana y tarde, así como por carencia de máquinas expendedoras, iluminación y seguridad.

\section{La Zona Metropolitana de Pachuca y su movilidad}

El intercambio de población que mantiene la Zona Metropolitana de Pachuca puede indicar incrementos de actividad económica en los municipios colindantes; es decir, un buen sistema de transporte no sólo beneficia a la ciudad o territorio donde se implanta, sino a toda una región o una macro-región. Analizar estos movimientos entre los municipios permite conocer su ubicación, primacía y atracción poblacional, el uso del transporte, el tiempo utilizado, el costo del servicio y la distancia recorrida (Aroca, 2007).

La ZMP se compone de siete municipios: Pachuca, Mineral de la Reforma, Mineral del Monte, San Agustín Tlaxiaca, Epazoyucan, Zapotlán de Juárez y Zempoala, según lo estipulado por el grupo interinstitucional (SEDESOL, CONAPO e INEGI, 2012).

En México, desde 1990, se ha observado un crecimiento sostenido de población en algunas ciudades; un ejemplo es el fenómeno urbano que se gesta en el centro del país y que encabeza la Ciudad de México. De acuerdo con datos de INEGI, las zonas urbanas de Hidalgo han crecido significativamente, principalmente la Zona Metropolitana de Pachuca, la cual ha pasado de tener una población de 276,512 en 1990 (INEGI, 1990) a 375,022 en el 2000 (INEGI, 2000), a 512,196 en 2010 (INEGI, 2010) y, finalmente, a 557,093 en 2015 (INEGI, 2015). La configuración de la población por edad y sexo permite tener un punto de partida para todo fenómeno demográfico, de manera que la distribución por sexo es de 47.2 y 52.8 para hombres y mujeres, respectivamente, en el último año analizado. 
Cuadro 1. Movilidad laboral y educativa de la Zona Metropolitana de Pachuca, 2015

\begin{tabular}{|c|c|c|c|c|c|}
\hline & \multirow[b]{2}{*}{ Municipios } & \multicolumn{2}{|c|}{ Movilidad laboral } & \multicolumn{2}{|c|}{ Movilidad educativa } \\
\hline & & Abs. & Rel. & Abs. & Rel. \\
\hline \multirow{8}{*}{ 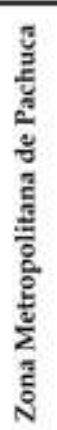 } & Epazoyucan & 2513 & $3.7 \%$ & 1397 & $4 \%$ \\
\hline & Mineral del Monte & 3001 & $4.4 \%$ & 1115 & $3 \%$ \\
\hline & Pachuca & 13723 & $20.2 \%$ & 6056 & $17 \%$ \\
\hline & Mineral de la Reforma & 32855 & $48.4 \%$ & 18044 & $50 \%$ \\
\hline & San Agustín Tlaxiaca & 6509 & $9.6 \%$ & 3232 & $9 \%$ \\
\hline & Zapotlán & 2439 & $3.6 \%$ & 1669 & $5 \%$ \\
\hline & Zempoala & 6834 & $10.1 \%$ & 4346 & $12 \%$ \\
\hline & Total & 67874 & $100.0 \%$ & 35859 & $100 \%$ \\
\hline
\end{tabular}

Fuente: elaboración propia con base en la Encuesta Intercensal, INEGI (2015).

De un total de 557,093 habitantes de la ZMP, alrededor de 67,874³ trabajan en otro municipio; es decir, uno de cada ocho personas; asimismo, 35,859 estudian fuera del municipio donde residen. Pachuca y Mineral de la Reforma presentan mayor movilidad laboral y educativa, lo cual puede responder a que se consideran municipios centrales (ver cuadro 1).

Cuadro 2. Origen-destino de la movilidad laboral de la Zona Metropolitana de Pachuca, 2015

\begin{tabular}{|c|c|c|c|c|c|c|c|c|c|c|c|c|c|c|}
\hline & Municipios & Epazoyuzan & $\begin{array}{l}\text { Mineral } \\
\text { de la } \\
\text { Reforms }\end{array}$ & $\begin{array}{c}\text { Mineral } \\
\text { del } \\
\text { Monte }\end{array}$ & $\begin{array}{l}\text { Panchuca } \\
\text { de } 5 \text { edve }\end{array}$ & $\begin{array}{c}\text { 5an } \\
\text { Agustin } \\
\text { Thaxiaca }\end{array}$ & $\begin{array}{l}\text { Znpoelain } \\
\text { de Juares }\end{array}$ & Zempoala & $\begin{array}{c}\text { Resto del } \\
\text { estado } \\
\text { de } \\
\text { Hidalgo }\end{array}$ & $Z C M$ & $\begin{array}{l}\text { Resto } \\
\text { del } \\
\text { pais }\end{array}$ & $\mathrm{NE}$ & Als. & Rel. \\
\hline \multirow{8}{*}{$\begin{array}{l}\text { Lugar de } \\
\text { residencia }\end{array}$} & Epazayucain & 0 & 134 & 12 & 131 & 0 & 0 & 111 & 122 & 183 & 23 & 106 & 842 & $1.20 \%$ \\
\hline & $\begin{array}{l}\text { Mineral de fla } \\
\text { Reforma }\end{array}$ & 239 & 0 & 16 & 1,758 & 119 & 63 & 263 & 2638 & 2969 & 263 & 132 & 8,460 & 12.30 \\
\hline & $\begin{array}{l}\text { Mineral del } \\
\text { Monte }\end{array}$ & s & 50 & 0 & 163 & 15 & 0 & 7 & 2001 & $7 \mathrm{~b}$ & 13 & 18 & 550 & $16.90 \%$ \\
\hline & $\begin{array}{l}\text { Pachoca de } \\
\text { Sola }\end{array}$ & 1,748 & 25,196 & 2,577 & 0 & 4,114 & 1,304 & 4,109 & 5,423 & 4,617 & 565 & 1,198 & 50,837 & 74.205 \\
\hline & $\begin{array}{l}\text { 5an Agustin } \\
\text { Tlaxiaca }\end{array}$ & 4 & 492 & 3 & 556 & 0 & It & 88 & 603 & 986 & 201 & 115 & 3,059 & $4.50 \%$ \\
\hline & $\begin{array}{l}\text { Zapottan de } \\
\text { luâre. }\end{array}$ & 0 & 164 & 1 & 68 & 201 & 0 & 44 & 466 & 387 & 24 & $\kappa 2$ & 1,437 & $2.10 \%$ \\
\hline & Zempoala & 42 & 200 & 6 & 208 & 16 & 74 & 0 & 1,068 & 1,353 & 308 & 170 & 3,328 & $4.90 \%$ \\
\hline & Als. & 2,041 & 26.336 & 2.615 & 2904 & 4,459 & 1,499 & 4.622 & 10,520 & 10,571 & 1,186 & 1.820 & 68,513 & $10000 \mathrm{~s}$ \\
\hline Total & Rel. & 37 & 383 & $4 \%$ & 48 & 78 & $2 \%$ & 75 & $15 \%$ & $15 \%$ & $2 \%$ & 32 & 160\%, & \\
\hline
\end{tabular}

Fuente: elaboración propia con base en la Encuesta Intercensal, INEGI (2015).

\footnotetext{
${ }^{3}$ Para identificar esta población, se recodificó primero la variable ZMP; una vez definida, se identifica a las personas que trabajan en el mismo municipio donde residen y las que trabajan en un municipio diferente.
} 
Cuadro 3. Origen-destino de la movilidad educativa de la Zona Metropolitana de Pachuca, 2015

\begin{tabular}{|c|c|c|c|c|c|c|c|c|c|c|c|c|c|c|}
\hline & \multirow[b]{2}{*}{ Municipios } & \multicolumn{13}{|c|}{ Lugar de estudio } \\
\hline & & Epazoyucan & $\begin{array}{c}\text { Mineral } \\
\text { de la } \\
\text { Reformat }\end{array}$ & $\begin{array}{c}\text { Mineral } \\
\text { del } \\
\text { Monte }\end{array}$ & $\begin{array}{l}\text { Pachuca } \\
\text { de Soto }\end{array}$ & $\begin{array}{c}\text { San } \\
\text { Agustin } \\
\text { Tlaxiaca }\end{array}$ & $\begin{array}{c}\text { Zapotian } \\
\text { de } \\
\text { Juárez }\end{array}$ & Zemposala & $\begin{array}{l}\text { Resto } \\
\text { del } \\
\text { estado } \\
\text { de } \\
\text { Hidalgo }\end{array}$ & $\mathrm{ZCM}$ & $\begin{array}{l}\text { Resto } \\
\text { del } \\
\text { pats }\end{array}$ & NE & Atre. & Rel. \\
\hline \multirow{7}{*}{$\begin{array}{l}\frac{5}{y} \\
\frac{y}{y} \\
\frac{y}{8} \\
y \\
y \\
y \\
y \\
y\end{array}$} & Epazoyucan & 0 & 147 & 0 & 876 & 2 & 0 & 96 & 35 & 5 & 0 & 236 & 4,182 & $12 \%$ \\
\hline & $\begin{array}{l}\text { Mineral de la } \\
\text { Reforma }\end{array}$ & 128 & 0 & 119 & 14,580 & 0 & i) & 498 & 1,345 & 341 & 141 & 892 & 48,817 & $136 \%$ \\
\hline & $\begin{array}{l}\text { Mineral del } \\
\text { Monte }\end{array}$ & 4 & 10 & 0 & 882 & 4 & 0 & 8 & 155 & 3 & 2 & 47 & 4,0N9 & $11 \%$ \\
\hline & $\begin{array}{l}\text { Pachuca de } \\
\text { Soto }\end{array}$ & a & 984 & 28 & 0 & 520 & 14 & 449 & 928 & 576 & 270 & 2,287 & 84,917 & $237 \%$ \\
\hline & $\begin{array}{l}\text { San Agustin } \\
\text { Tlaxiaca }\end{array}$ & a & 44 & 3 & 2,049 & 0 & 174 & 80 & 427 & 175 & 27 & 253 & 10,780 & $30 \%$ \\
\hline & $\begin{array}{l}\text { Zapotián de } \\
\text { Juárez }\end{array}$ & a & 29 & 2 & 1,044 & 100 & i) & 33 & 161 & 19 & 17 & 264 & 5,496 & $15 \%$ \\
\hline & Zemposala & 174 & 118 & 12 & 2870 & 35 & 66 & 0 & 467 & 94 & 30 & 480 & 13,295 & $37 \%$ \\
\hline \multirow[t]{2}{*}{ 'Total } & Abs. & 306 & 1,332 & 164 & 22,301 & 661 & 254 & 1,164 & 3,518 & 1,213 & 487 & 4,459 & 35,859 & $478 \%$ \\
\hline & Rel. & $1 \%$ & $4 \%$ & $0 \%$ & $62 \%$ & $2 \%$ & $1 \%$ & $3 \%$ & $10 \%$ & $3 \%$ & $1 \%$ & $12 \%$ & $100 \%$ & \\
\hline
\end{tabular}

Fuente: elaboración propia con base en la Encuesta Intercensal, INEGI (2015).

Con relación al destino de la movilidad, el 38\% trabaja en el municipio de Mineral de la Reforma; éste ocupa el lugar número 1 en atracción de población; el 15\% labora en la Zona Centro y el resto en el estado de Hidalgo, lo cual implica una constante movilidad urbana al interior y exterior de la Zona Metropolitana de Pachuca (ver cuadro 2).

Llama la atención el número de personas que se mueve a los estados de la zona centro del país, es decir, entre Puebla, Tlaxcala, Morelos, Querétaro y Estado de México, donde intrínsecamente se observa la existencia de mercados de trabajos locales, los cuales explican los movimientos de población dentro y fuera del estado, aunado a la dispersión y desconcentración del mercado laboral de la Ciudad de México y, por ende, al surgimiento de mercados de trabajo atractivos para personas de la ZMP. El dato de las personas que viajan al resto del país a trabajar parece que no es relevante, pues sólo el $2 \%$ reside en la ZMP pero trabaja en un estado diferente a Hidalgo.

La movilidad por fines educativos también es un elemento importante; de acuerdo con datos de la Encuesta Intercensal INEGI (2015), a nivel nacional, alrededor de 3.1 millones de personas estudian en un municipio diferente al que residen; los datos por sexo son muy similares con $50.1 \%$ para los hombres y $49.9 \%$ para las mujeres. En el caso de la Zona Metropolitana de Pachuca, el 62\% estudia en el municipio de Pachuca; el 10\%, en un municipio del resto del estado; y el 4\%, en Mineral de la Reforma; esto debido a la ubicación de los principales centros educativos en Pachuca, la capital del estado; por ello, le da la centralidad en este aspecto (ver cuadro 3). 
Asimismo, es importante conocer cuál es el medio de transporte que las personas de la ZMP utilizan en sus traslados a su trabajo. De acuerdo con el INEGI (2015), el 45.8\% utiliza camión, taxi, combi o colectivo; el 29.3\% usa vehículo particular (automóvil, camioneta o motocicleta); el 17.7\% se desplaza caminando; el 2.4\% emplean trasporte laboral. Respecto al traslado de las personas para estudiar, el 39.9\% ocupa camión, taxi, combi o colectivo; le sigue el uso de vehículo particular (automóvil, camioneta o motocicleta) con el $21.0 \%$; y el $36.9 \%$ lo hace caminando.

Para la movilidad tanto laboral como educativa, los principales medios de transporte son los públicos, es decir, los utiliza alrededor del $40 \%$ de la población; por ello, es importante estudiar estos medios como fundamentales dentro del fenómeno de la movilidad dentro de la ciudad.

Los datos para la Zona Metropolitana de Pachuca indican que, de las personas que se trasladan por motivos educativos, el $48.2 \%$ corresponde a hombres y el $51.8 \%$ a mujeres, con una concentración de un $62.7 \%$ en las edades de $13-18$ años, seguido del $35.4 \%$ de 19 a 35 años, y, finalmente, el 1.7\% de 36 a 59 años (ver gráfico 1).

Gráfico 1. Porcentaje de movilidad educativa por grupos de edades de la ZMP, 2015

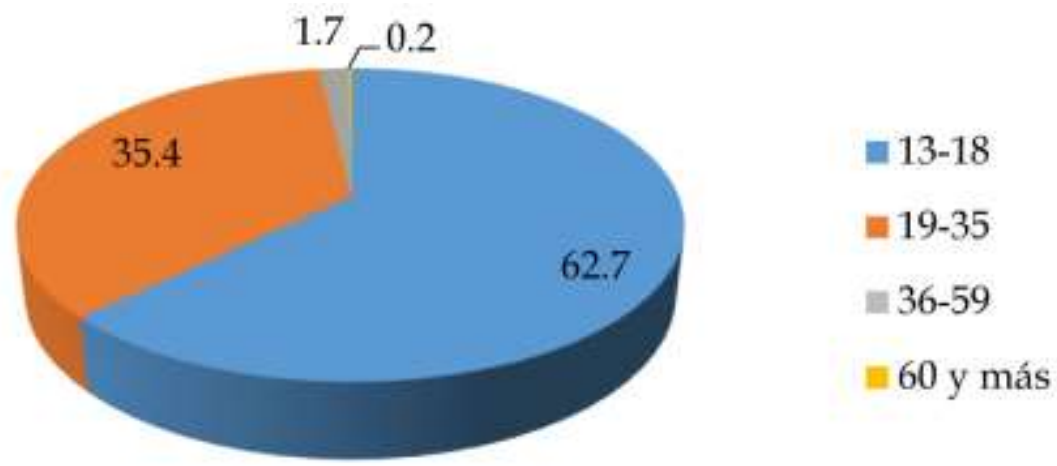

Fuente: elaboración propia con datos de la Encuesta Intercensal, INEGI (2015).

Respecto a la movilidad laboral, el 55.6\% corresponde a hombres y el $44.4 \%$ a mujeres; se observa la prevalencia masculina, pese a la incorporación femenina en el mercado laboral, lo cual puede indicar que ellas trabajan en lugares más locales que les implica moverse menos. En cuanto a la edad, el $47.5 \%$ tiene de 19 a 35 años; el $45.5 \%$ se encuentra entre 36 y 59 años; el 3\% y el 4\% corresponden a personas de 13 a 18 años y de 60 y más, respectivamente (ver gráfico 2). 
Gráfico 2. Porcentaje de movilidad laboral por grupos de edades, de la ZMP, 2015

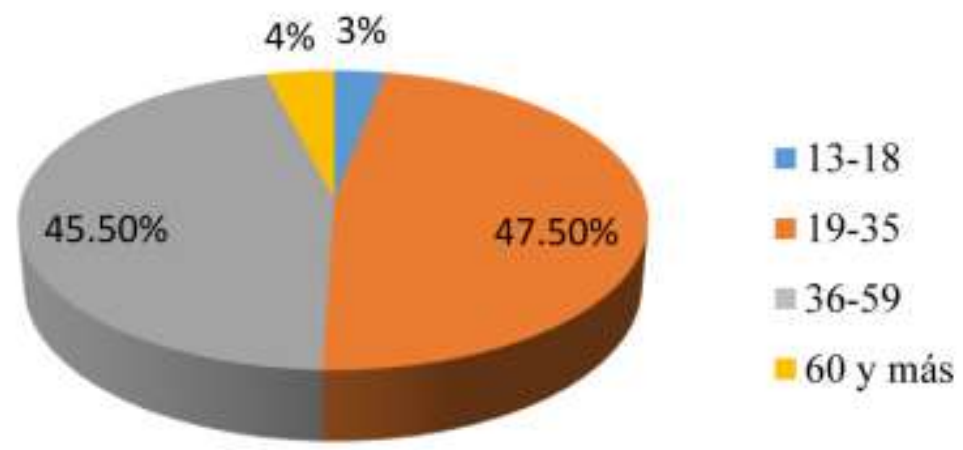

Fuente: elaboración propia con datos de la Encuesta Intercensal, INEGI (2015).

El sistema de transporte de la ciudad de Pachuca es semi radial concéntrico, es decir, implica una conexión de las vialidades hacia el centro, las cuales se conforman por enlaces de tipo federal y estatal (Municipio de Pachuca, 2016). Debido a las características topográficas y al crecimiento poblacional, se ha conformado un sistema vial que une el norte y el sur de la ciudad y converge en el centro histórico de la mancha urbana (ver mapa 1).

Las vialidades de la ciudad tienen una orientación hacia el sur del municipio de Pachuca, y de este al oeste, con una trama urbana irregular proveniente de la época colonial donde se dio un importante proceso minero, además de lo angosto de las calles, lo cual ha ocasionado que se sature la zona central. Por tanto, se carece de una estructura que comunique eficaz y eficientemente a toda la ciudad.

El sistema de transporte de la Zona Metropolitana no se encuentra organizado, planeado ni integrado a las necesidades de la sociedad; prueba de esto son los recorridos que permean hacia el centro de la ciudad sin ninguna conexión entre ellos llegando a realizarse triangulaciones, transbordos y recorridos innecesarios para llegar a sus destinos, lo cual incrementa tiempos y distancias. Antes de la implementación del Tuzobús, los traslados se realizaban en rutas de combis, camiones o taxis que conectaban la ciudad e involucraban problemas más importantes, como la informalidad, los bajos estándares de calidad y la degradación física de la flota vehicular. Así, la vida cotidiana estaba enmarcada por recorridos en malas condiciones de movilidad, donde no se regulaba la calidad del servicio; sin embargo, esta situación aún perdura, pues el crecimiento que ha mantenido la ciudad no corresponde con una red completa y ordenada de transporte. 
Mapa 1. Tipos de vialidades de la Zona Metropolitana de Pachuca, 2018

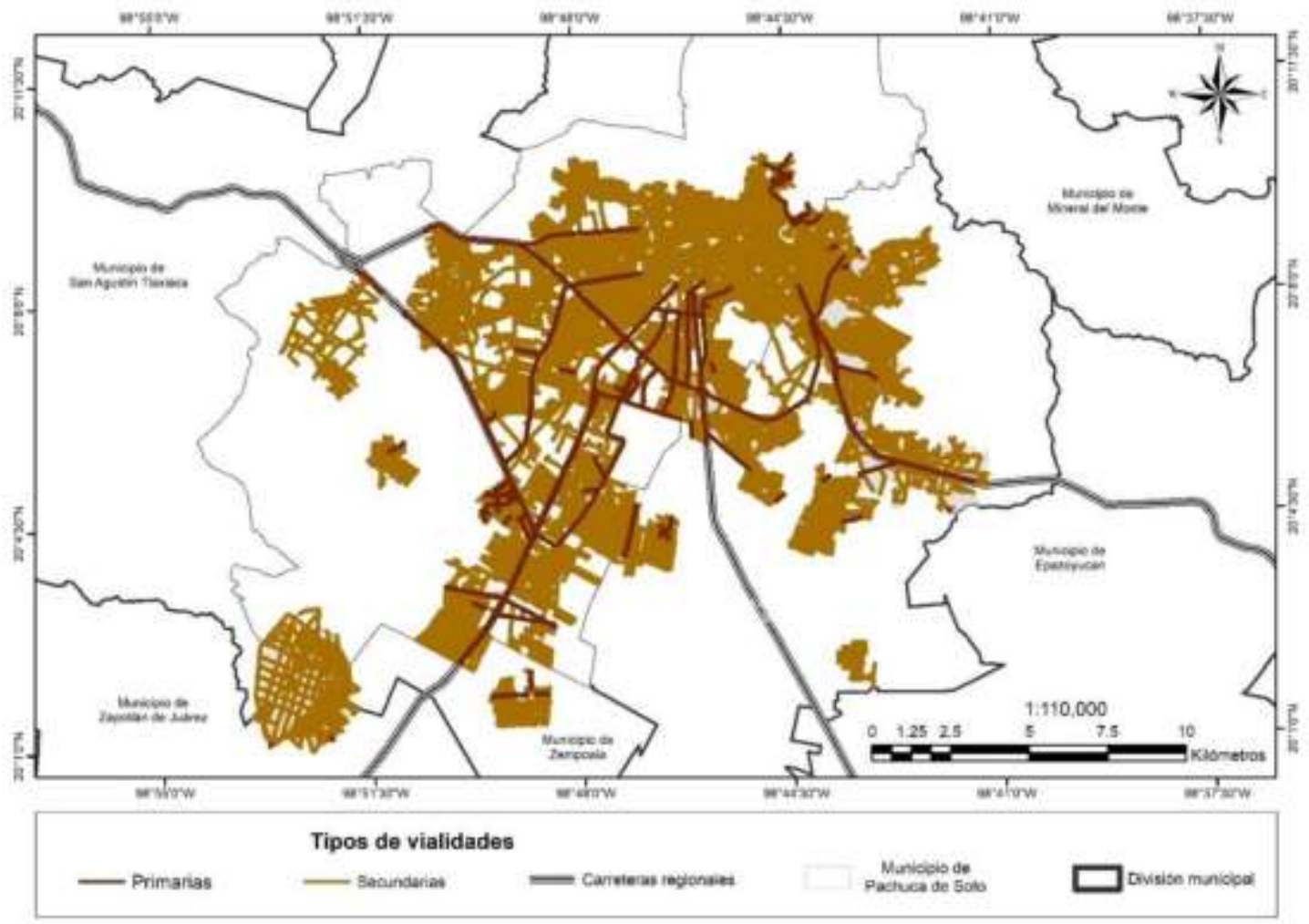

Fuente: elaboración propia con base en INEGI (2020).

Actualmente, la movilidad se sustenta en transporte público (Tuzobús) coordinado por el gobierno local, transporte público concesionado a empresas privadas (microbuses, unidades tipo van, taxis) y transporte privado (automóvil). El público se integra por taxis, combis y el Tuzobús; este último es un Sistema Integrado de Transporte Masivo puesto en marcha el 16 de agosto de 2015 en la ciudad de Pachuca y su Zona Metropolitana, cuyos beneficiarios son alrededor de 2'701,618 personas mensuales, 675,405 semanales y 108,109 diarios (ver cuadro 4) (Gobierno del Estado de Hidalgo, 2018); su objetivo es mejorar la calidad y la eficiencia de los traslados, lo cual no se ha cumplido debido al gasto del tiempo en movilizarse y a la economía de los usuarios. 
Cuadro 4. Pasajeros promedio del transporte masivo Tuzobús, 2018

\begin{tabular}{lccc}
\hline Pasajeros promedio & Total en el sistema & Adulto mayor & Discapacitado \\
\hline Día & 108,109 & 3,750 & 315 \\
Semana & 675,405 & 23,062 & 1,937 \\
Mes & $2^{\prime} 701,618$ & 92,250 & 7,749 \\
\hline
\end{tabular}

Fuente: Secretaría de Movilidad y Transporte de Hidalgo (2018).

Las rutas con las que cuenta son la troncal (Téllez-Centro), integrada por 32 estaciones que cruzan y dividen en dos la ciudad de Pachuca, ubicándose en un carril del lado izquierdo de la vialidad; esta ruta hace traslados en el parador que va deteniéndose en cada una de las estaciones y la exprés, la cual se para sólo en algunas estaciones definidas; además, cuenta con 26 rutas alimentadoras (ver mapa 2), cuyo propósito es hacer un transporte más eficiente (Gobierno del Estado de Hidalgo, 2018).

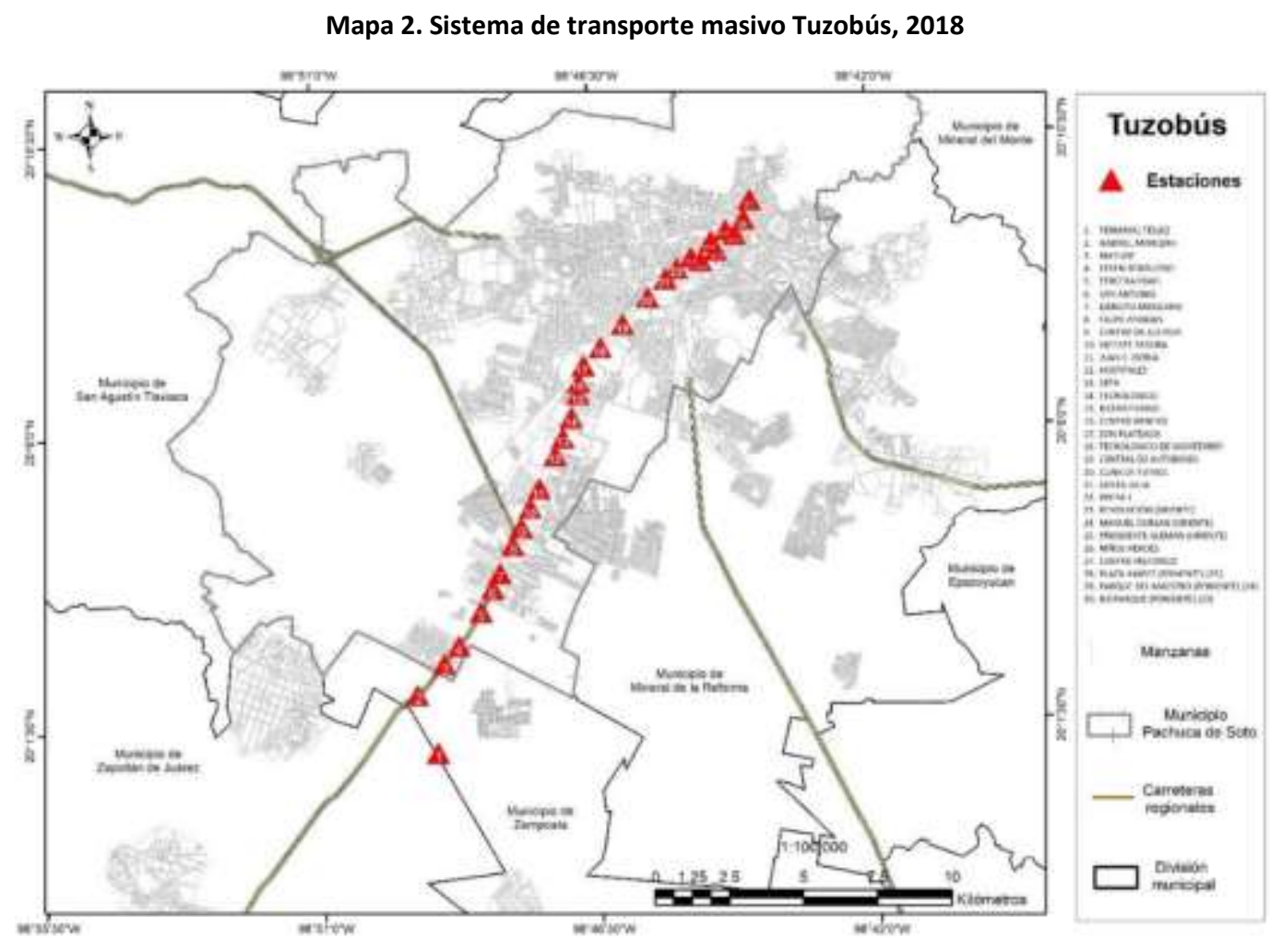

Fuente: elaboración propia con datos de Marco Geoestadístico, INEGI (2010). 
De acuerdo con los datos proporcionados por la Encuesta Nacional de Calidad e Impacto Gubernamental (ENCIG) de INEGI, se señala que cuatro de cada diez usuarios califican insatisfactoriamente el servicio proporcionado por el Tuzobús.

Dentro de la encuesta se evaluó el nivel de satisfacción de los usuarios de los servicios de autobús de tránsito rápido de Jalisco-Macrobús, Baja California-SITT3, Estado de México-Mexibús, Guerrero-Acabús e Hidalgo-Tuzobús. Los usuarios de este último en Pachuca son los menos satisfechos por este tipo de transporte. Para el análisis de la eficiencia del Tuzobús, se encuestó a 138,141 usuarios, donde alrededor del 42\% expresó estar insatisfecho con este tipo de transporte; además, seis de cada diez dijeron que son insuficientes las rutas.

Antes del Tuzobús los traslados en transporte público eran un poco más rápidos, yo vivo en San Antonio y tomaba la micro al centro y en media hora llegaba, ahora es mucho más complicado pues tengo que esperar la alimentadora y después esperar a que pase el Tuzobús y luego se llenan rápido y la espera es mucho más larga, es horrible (Gutiérrez, entrevista, 11 de julio de 2018).

Además, el 97.7\% dijo que hay ascenso de pasajeros en paradas oficiales; seis de cada diez señalaron que los horarios de corridas están disponibles en las estaciones y el 63\% afirmó que transcurre poco tiempo entre una unidad y otra.

No cubren la periferia de la mancha urbana, las rutas perjudican la movilidad de las personas, pues alargan los trayectos de un lugar a otro; el servicio no es tan eficiente, pues las bases están retiradas entre si y no hay el servicio del operador y el usuario. Los operadores no esperan a que las personas aborden, pasan muy rápido, ya que el tiempo es de dos minutos (Vite, entrevista, 5 de septiembre de 2018).

También seis de cada diez señalan que el espacio del Tuzobús es confiable y seguro para viajar, con unidades en buen estado y funcionales. De acuerdo con INEGI, en Hidalgo, el índice de satisfacción de los usuarios del transporte público (tipo autobús urbano, van, combi o microbús) es bajo, pues se ubica en un 45\% de aprobación. Esto coincide con la información recabada en campo, donde nueve de cada diez usuarios manifestaron su descontento con la implementación de este tipo de transporte.

Existen rutas del transporte público que no están vinculadas directamente con el Tuzobús pero fueron modificadas, haciendo gastar más tiempo y dinero a los usuarios. Este incremento de los costos se atribuye en buena parte al sistema de explotación del servicio basado en la rentabilidad económica, lo cual deja de lado el carácter de servicio público que debiera tener añadiéndole otros elementos, como el tiempo de traslado, esperas e incomodidad. 
Me veo obligada a adquirir una tarjeta para transbordar de 17 pesos, y de no contar con ella se tiene que hacer doble pago por cada transbordo (Martínez, entrevista, 5 de septiembre de 2018).

Me parece fue muy buena idea el querer mejorar el sistema de transporte, pero no se ha logrado aún, tal vez más adelante, pero no ayuda hacer transbordos, pierdo mucho tiempo esperando que llegue la alimentadora y gasto más que antes (Santos, entrevista, 9 de octubre de 2018).

Además, el cambio de transporte de rutas de combis a Tuzobús ha implicado afectación en los sectores más vulnerables de la población (adultos mayores, personas con capacidades diferentes, etcétera), lo cual ha mermado su economía y también su posibilidad de acceso, ya que las paradas están mal ubicadas: unas están muy juntas y otras muy separadas.

Siempre he viajado de la colonia del ISSSTE hacia la central de abastos que es donde he trabajado desde hace 5 años, me iba en las combis grandotas que llegaban a la central de autobuses y se iban hasta el Hospital General, a lo mucho en 10 minutos estaba en el trabajo, ahora no me tengo que ir antes para poder llegar, por lo menos 30 minutos para llegar a mi trabajo, además el usar la alimentadora siempre hace más tardado el traslado (Morales, entrevista, 6 de septiembre de 2018).

No creo sea hoy un transporte eficaz, nos hemos acostumbrado, pero para llegar a la chamba debo ir con tiempo, pues he llegado tarde y me han descontado el día, antes era más rápido (Torres, entrevista 8 de octubre de 2018).

Tras la puesta en marcha del Tuzobús, las personas que no cuentan con automóvil privado y que viven muy alejadas de sus lugares de trabajo, estudio, entre otros, se han visto seriamente afectadas en su calidad y modos de vida al disminuir notoriamente la oferta de transporte público con que contaban y que conectaba sus lugares de residencia con los espacios de la ciudad donde realizan sus actividades cotidianas. En este sentido, la oferta de transporte público actual ha limitado, en gran medida, el acceso que tenían los individuos al mercado de trabajo, a las oportunidades de estudio y a las redes sociales que se encuentran más allá del entorno próximo, al dificultar los desplazamientos cotidianos debido a los problemas existentes en la cobertura horaria y espacial del sistema (Jouffe y Lazo, 2010).

Es un problema, parece que nunca lo planearon los fines de semana para entrar o salir de la ciudad es un caos mucho tráfico, nos quitaron un carril para el Tuzobús, según para mejorar el sistema de transporte y la realidad es otra, por lo menos 20 minutos más y no se diga para el centro ya ni ganas dan de ir (Ramírez, entrevista, 6 de septiembre de 2018).

He tenido varias dificultades hay que salir de casa con más tiempo de anticipación que antes que existiera el Tuzo, usar la alimentadora que no siempre llega en tiempo y todo eso te retrasa y en las mañanas lo hace más difícil para llegar a tiempo a clases, antes había varias opciones de transporte, creo nunca debieron quitarlas (Ramos entrevista, 7 de octubre de 2018). 
Sin embargo, la implementación de este transporte ha afectado no sólo a los usuarios de este transporte, sino también a los automovilistas que transitan sobre la vía donde corre el Tuzobús, pues se observan calles donde sólo existen dos carriles, incluso, aquellas que son más amplias, presentan un tráfico intenso originado por la reducción de carriles.

\section{Conclusiones}

En la actualidad, se han presentado constantes problemas del sistema de transporte metropolitano, como: informalidad, bajos estándares de calidad y degradación física de la flota vehicular, recorridos en malas condiciones de movilidad, carencia de una red completa de transporte, entre otros.

La ordenación territorial y urbanística del espacio debe considerar proyectos en que las redes de comunicación y transporte permitan distintas maneras de movilidad. El diagnosticar cómo se encuentra la movilidad cotidiana de la ciudad de Pachuca permite establecer un nuevo orden, más racional y equitativo. Por ello, es imperante la intervención de los gobiernos federal y municipal, así de la ciudadanía, a fin de que conjuntamente busquen mejorar la movilidad de la ciudad.

Este documento permite conocer el sistema de transporte y su apreciación en la Zona Metropolitana de Pachuca, la cual se encuentra en pleno proceso de desarrollo; a través del análisis de la información estadística y la percepción de los usuarios, se refleja un considerable atraso, manifestado en carencias en la cobertura, eficiencia, calidad, tiempos de traslado, etcétera, que, evidentemente, afectan a la población en general. Además, debido al crecimiento significativo de la población y a la conurbación con otros municipios, esta situación aumenta y se magnifica; por ello, es urgente una planeación del transporte que permita una adecuada movilidad urbana dentro de la ZMP.

Derivado de ello, en Pachuca se implantó el sistema de transporte denominado Tuzobús, cuyo objetivo es mejorar la manera de desplazarse de los habitantes de la ZMP y disminuir la congestión y la contaminación vehicular; sin embargo, esta modificación terminó afectando negativamente la vida cotidiana de ciudadanos, ya que no cumplió con su cobertura y ha desencadenado problemas en los desplazamientos de la población impactando a los sectores más vulnerables de la sociedad, pues les ha representado mayor gasto en tiempo y dinero.

Asimismo, la movilidad urbana desarrollada en la ZMP enmarca un círculo vicioso, ya que algunos sectores de la población se han visto obligados a usar un medio de transporte privado, lo cual provoca conflictos de tránsito por el colapso de vías de comunicación; por lo tanto, los usuarios son los más afectados en cuanto a pérdidas económicas y de tiempo. 


\section{Referencias}

Alcántara, E. (2010). Análisis de la movilidad urbana espacio, medio ambiente y equidad. Bogotá Colombia: Corporación Andina de Fomento CAF.

Arango, A. (2010). La periferia conurbada de la Ciudad de México:Movilidad cotidiana y manejo de tiempo de la población en unidades habitacionales de Ixtapaluca. Verteidigung.

Aroca, P. (2007). Impacto sobre el crecimiento regional de la migración y conmutación interregional en Chile. Santiago de Chile, Chile.

Avellaneda, P., y Lazo, A. (2011). Aproximación a la movilidad cotidiana en la periferia pobre de dos ciudades latinoamericanas los casos de Lima y Santiago de Chile. Revista transporte y territorio(4), 47-58.

Bazant, J. (2018). Movilidad y planeación urbana estratégica. Ciudad de México: Trillas.

Ciudadanía metropolitana. (2018). Observatorio de movilidad urbana. Recuperado de: http:/ / ciudadaniametropolitana.org.ar/2018/09/hacia-una-movilidad-sustentable/

Comisión Asesora Presidencial Pro Movilidad Urbana. (2014). Probleas de la movilidad urbana: estrategias y medidas para su mitigación. Santiago de Chile.

Cruz-Muñoz, F. (2018). La movilidad urbana: dimensiones y desafíos. EURE, 44(133), 1-6. Recuperado de: http:/ / www.redalyc.org/articulo.oa?id=19656548013

Gobierno del Estado de Hidalgo. (2018). Sistema Integrado de Transporte Masivo de Hidalgo. Recuperado de: http:/ / sitmah.hidalgo.gob.mx/?p=557

Gutiérrez, J. y García, J. (2005), Movilidad por motivo de trabajo en la comunidad de Madrid. Madrid: Universidad Complutense de Madrid

INEGI. (1990). Censos de Población y Vivienda 1990. México: INEGI.

INEGI. (2000). Censo de Población y Vivienda 2000. México: INEGI.

INEGI. (2010). Censo de Población y Vivienda 2010. México: INEGI.

INEGI. (2015). Encuesta Intercensal 2015. México: INEGI.

INEGI. (2017). Encuesta Nacional de Calidad e Impacto Gubernamental, ENCIG 2017. México: INEGI.

ITDP. (2013). Hacia una estrategia nacional integral de movilidad urbana. Ciudad de México: Instituto de Políticas para el transporte $y$ el Desarrollo. Recuperado de: http://mexico.itdp.org/wpcontent/uploads/Movilidad-Urbana-Sustentable-MUS_.pdf

Jans, M. (2017). Movilidad urbana: En camino a sistemas de transporte colectivo integrados. Revista AUS, 6-11. Recuperado de: http://revistas.uach.cl/index.php/aus/article/view/616

Jirón Martínez, P. (2015). La movilidad como oportunidad para el desarrollo urbano y territorial. En E. U. Valparaíso (Ed.), La ciudad que queremos. Santiago de Chile: Biblioteca del Congreso Nacional de Chile.

Jouffe, Y., y Lazo, A. (2010). Las prácticas cotidianas frente a los dispositivos de la movilidad: Aproximación política a la movilidad cotidiana de las poblaciones pobres periurbanas de Santiago de Chile. EURE, $29-47$.

Karakayaci, Z. (2016). The concept of urban sprawl and its causes. Journal of International Social Research(9), 815815.

Korsu, E. (2010). La proximité dolicile-travail dans les choix residentielles et professinnels de I’individu hyper moderne. En M. Massot, Mobilités et modes de vie metropolitaines. France.

Mendoza, J. y García, J. (2013). Las iniciativas innovadoras del Gobierno del Estado de Hidalgo: El caso del Sistema Bus Rapid Transit (BRT) "Tuzobús" en la Ciudad de Pachuca de Soto. Pachuca: Plaza y Valdes-UAEH.

Municipio de Pachuca. (2016). Plan Municipal de Desarrollo Pachuca de Soto 2016-2020. Pachuca: Gobierno del estado de Hidalgo.

Negrete, M. (2018). El desafío de la movilidad y el transporte urbano metropolitano . México: El Colegio de México.

ONU-HABITAT. (2020). ONU-HABITAT. Recuperado de: https://onuhabitat.org.mx/index.php/folleto-dedatos-poblacionales-2020 
Pérez, R. (2015). De la flânerie al tránsito peatonal: la negación del derecho a la ciudad. Cybergeo : European Journal of Geography, Espace, Société, Territoire, 724. Recuperado de: http://journals.openedition.org/cybergeo/26991 ; DOI : https:/ /doi.org/10.4000/cybergeo.26991

Secretaría de Gobernación. (2020). Plan Nacional de Desarrollo 2019-2024. Diario Oficial de la Federación Recuperado de: https://www.dof.gob.mx/nota_detalle.php?codigo=5565599yfecha=12/07/2019

Secretaría de Movilidad y transporte de Hidalgo. (2018). Manifestación de impacto de Hidalgo. Recuperado de Gobierno del Estado de Hidalgo: http://transparencia.hidalgo.gob.mx/descargables/dependencias/obrasp/Fraccion\%2028/Comunic aciones/2015/26\%202015C265\%20Y\%20059\%20MANIFIESTO\%20DE\%20IMPAC.\%20AMB.\%20TUZ OBÚS/MANIFIESTO\%20TUZOBÚS.pdf

SEDESOL,CONAPO e INEGI. (2012). Delimitación de las Zonas Metropolitanas de México. México.

Suárez, M., y Delgado, J. (2010). Patrones de movilidad residencial en la Ciudad de México como evidencia de co-localización de población y empleos. EURE, 30(107), 67-91. Recuperado de: https://www.scielo.cl/scielo.php?script=sci_arttext\&pid=S0250-71612010000100004

Vadillo, C. (2019). Movilidad en las metropolis. Ciudad de México, México: Siglo veintiuno editores.

Zamora Colín, U., Campos Alanís , H., y Calderón Maya, J. R. (2013). Bus Rapid Transit (BRT) en ciudades de América Latina, los casos de Bogotá (Colombia) y Curitiba. Quivera. Revista de Estudios Territoriales, 15(1),101-118. Recuperado de: https:/ / www.redalyc.org/articulo.oa?id=40128395007

\section{Entrevistas}

Gutiérrez Hernández, C. (2018). Percepción del transporte masivo Tuzobús, entrevistado por el autor, Pachuca de Soto Hidalgo, 11 de julio 2018.

Martínez López, J. (2018), Percepción del transporte masivo Tuzobús, entrevistado por el autor, Pachuca de Soto Hidalgo, 5 de septiembre de 2018.

Morales Robles, R. (2018), Percepción del transporte masivo Tuzobús, entrevistada por el autor, Pachuca de Soto Hidalgo, 6 M. septiembre de 2018.

Ramírez Canales, María (2018), Percepción del transporte masivo Tuzobús, entrevistado por el autor, Pachuca de Soto Hidalgo, 6 de septiembre de 2018.

Ramos Marroquín, A. (2018), Percepción del transporte masivo Tuzobús, entrevistado por el autor, Pachuca de Soto Hidalgo, 11 de octubre de 2018.

Santos López, J. (2018). Percepción del transporte masivo Tuzobús, entrevistado por el autor, Pachuca de Soto Hidalgo, 7 de octubre de 2018.

Torres Olmedo, F. (2018). Percepción del transporte masivo Tuzobús, entrevistado por el autor, Pachuca de Soto Hidalgo, 8 de octubre de 2018.

Vite Melo, A. (2018). Percepción del transporte masivo Tuzobús, entrevistado por el autor, Pachuca de Soto Hidalgo, 5 de septiembre de 2018. 